In this case the pellet penetrates just past mid-radius resulting immediately in a hollow density profile. The profile fills in over $200 \mathrm{~ms}$ and recovers to a peaked profile in $\cong 400 \mathrm{~ms}$ before the density begins to decay.

The temporal evolution of other plasma parameters after pellet injection is shown in Fig. 4. Concomitant with the rise in the density is a sharp fall in the electron and ion temperatures. Recovery begins immediately with the density slowly falling and the temperature rising but on a faster time scale such that there is a net increase in plasma energy that peaks some $700 \mathrm{~ms}$ after injection. The effect on the impurity content is that $Z_{\text {eff }}$ is reduced from 2.5 down to 1.6 , agreeing well with the value calculated from simple dilution of the existing plasma by the injection of pure deuterium. The neutron yield (D-D reaction rate) also increases in close agreement with the rise in deuterium density and temperature.

(c) On a longer time scale the net effect of the pellet is to produce a clean, high density plasma that lasts for $\cong 0.5$ - $1 \mathrm{~s}$ after injection before most parameters return approximately to their prepellet values. Under certain plasma conditions the effect of the pellet is longer lived. The application of high power ICRF heating to such a centrally peaked

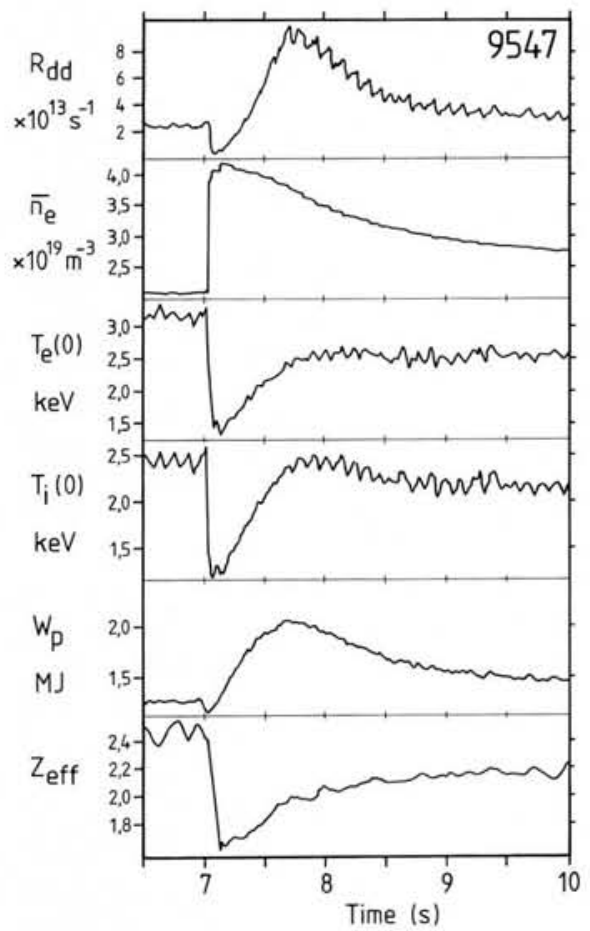

Fig. 4 - Temporal evolution of several plasma parameters after pellet injection. The $D-D$ reaction rate $R_{d d}$, average electron density $\bar{n}_{e}$, central electron and ion temperatures $T_{e}$ and $T_{i}$, total plasma energy content $W_{p}$ and the impurity content $Z_{\text {eff }}$. This is a 3 MA discharge with no auxiliary heating, the pellet was injected at $7 \mathrm{~s}$.

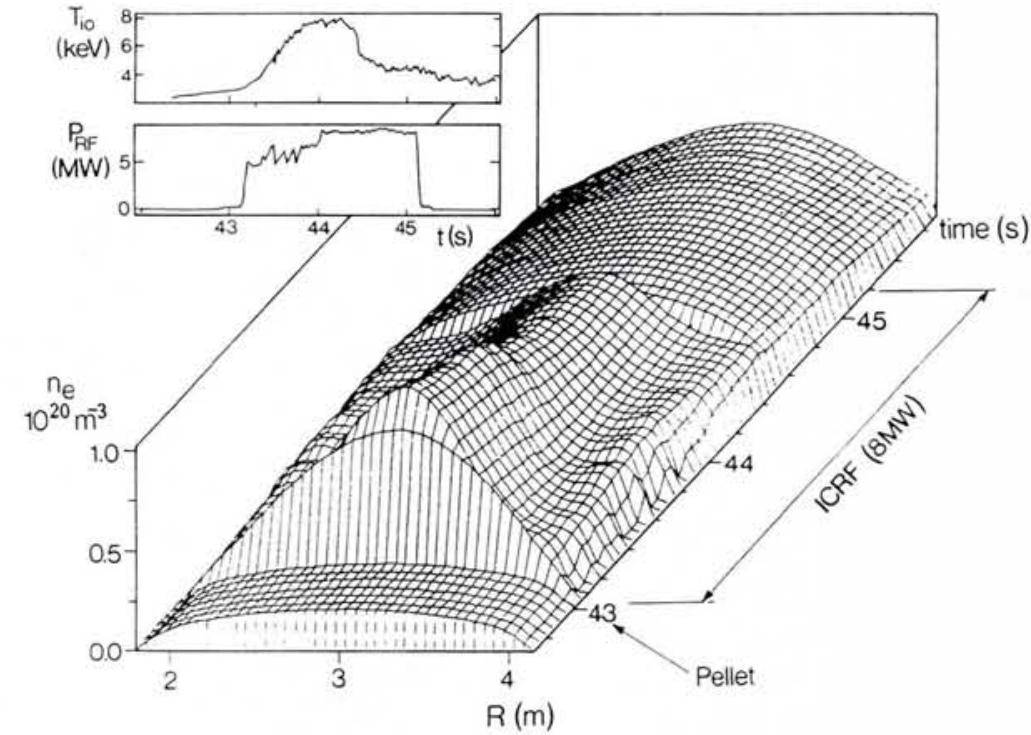

Fig. 5 - Temporal and spatial evolution of the electron density after pellet injection to the centre of the plasma followed immediately by high power ICRF heating. Also shown are the temporal evolution of the ion temperature and the ICRF input power.

density profile (Fig. 5), obtained when the pellet penetrates to the centre of the plasma, results in extremely high electron and ion temperatures and, coupled with the high deuteron concentration produces a greatly enhanced fusion neutron yield.

\section{Conclusions}

The results of recent experiments on JET have demonstrated the efficacy of pellet fuelling of high temperature plasmas. The direct fuelling of the core produces a pure, dense plasma with a centrally peaked density profile. The application of auxiliary heating to such plasmas can produce a long lived ( $>1 \mathrm{~s}$ ) dense, hot core giving a high fusion yield. Further work will concentrate on maintaining and extending the life time of these plasma conditions. Developmental work will continue on injector technology to increase the pellet speed for effective penetration of fusion temperature plasmas.

\section{Acknowledgment}

The author would like to acknowledge A. Gondhalekar and P. Kupschus for their collaboration and helpful comments, the pellet injection and ICRH groups at JET and G. Schmidt and the US pellet injection team, for supplying the data.

\section{FURTHER READING}

Details of the JET Pellet Injector Milora S et al., Proceedings of the IEEE Symposium on Fusion Engineering, Monterey, October $1987 \mathrm{Vol}$. 2, page 784.

Kupschus P. et al., ibid Vol. 2, page 780.

Advances in High Speed Pellet Development Sonnenberg K. et al., ibid Vol. 2, page 1208.

\section{Experimental Results}

Gondhalekar A. et al., Proc. of the 11th IAEA Conf. on Plasma Physics and Controlled Nuclear Fusion Research, Kyoto 1986 Vol. 3, page 457.

Kupschus P. et al., Proc. 15th European Conf. on Controlled Fusion and Plasma Heating, Dubrovnik, May 1988 Vol. 12b, part 1, page 143.

Milora S. et al., ibid, page 147.

\title{
Physical Society of Japan
}

The 44th Annual Meeting of the Physical Society of Japan is to be held at Tokai University, Hiratshuka-shi, Kanagawa-ken from 28-31 March 1989. Application deadline for presentation of papers is $14 \mathrm{Dec}$. 1988. As the Society is a Collaborating Society of EPS, IOMs enjoy the same rights as members of the PSJ. Enquiries should be addressed to:

The Physical Society of Japan Room 211, Kikai-Shinko Building 3-5-8 Shiba-Koen, Minato-Ku Tokyo 105, Japan Tel.: (3) 4342671
Through the collaboration agreement concluded between EPS and the PSJ in 1987, Individual Ordinary Members of EPS may also subscribe to the Journal of the Physical Society of Japan at the same rate as members of the PSJ.

For Volume 58 (1989) the annual rate for Europe is: $Y 14000$ surface mail

$$
Y 39000 \text { airmail }
$$

Questions regarding subscriptions should be sent to the Physical Society of Japan at the address given in the adjacent column. 